\title{
A STUDY ON GROWTH CO-EFFICIENT AND RELATIVE CONDITION FACTOR OF THE MAJOR CARP (CATLA CATLA) IN TWO LAKES DIFFERING IN WATER QUALITY
}

\author{
K.L. SACHIDANANDAMURTHY- H.N. YAJURVEDI ${ }^{*}$ \\ Department of Zoology, University of Mysore, Manasagangotri, Mysore-570 006, India \\ (phone: 0821 2419778) \\ *Corresponding author \\ e-mail: hnyajurvedi@yahoo.co.in \\ (Received $26^{\text {th }}$ July 2006 ; accepted $16^{\text {th }}$ January 2008)
}

\begin{abstract}
Physico-chemical water quality parameters viz. temperature, $\mathrm{pH}$, alkalinity, turbidity, total solids, DO, BOD, nitrite, nitrate, phosphate, ammonia, and hydrogen sulfide coupled with diversity and density of plankton were studied during different seasons (monsoon, winter, summer) for two consecutive calendar years in two lakes to find out whether differences in water quality are reflected in growth coefficient and relative condition factor of the major carp, Catla catla. Significantly higher levels of nutrients (phosphate, nitrate, nitrite) and ammonia, low plankton diversity, and significantly higher density of cyanophyceae were found in Yennehole lake (YL) compared to Belikere lake (BL). In addition, desmids were conspicuous by their absence in YL in contrast to their presence in BL. Concomitantly, growth factor (b) and relative condition factor $(\mathrm{Kn})$ of C.catla were $<3$ and $<1$ respectively in YL whereas they were close to 3 (or $>3$ ) and $>1$ respectively in BL in all the seasons in during the study period. The contrasting $\mathrm{b}$ and $\mathrm{Kn}$ values of the same species in two lakes differing in water quality provide an evidence that deterioration in water quality though not enough to cause death of fish, does adversely affect growth and well being of fish.
\end{abstract}

Keywords: Fish growth, water quality, Catla catla, relative condition factor, Algal bloom.

\section{Introduction}

There are good numbers of studies on seasonal variation in water quality of lakes (Banerjea 1967, Munawar 1970, Boyd 1979, Singh \& Rai 1984, Ansari \& Prakesh 2000, Sharma and Rathore 2000, Khabade et al. 2002, Datta et al. 2005, Sukamarn \& Das 2005). On the other hand there are studies on growth and well being of fish in water bodies (Le'Cren 1951, Jhingran 1952, 1968, Tesch 1968, Doha \& Dewan 1967, Botros 1970, Bashirulla \& Kader 1970, Siddique 1977, Quadri \& Mir 1980, Olatunde 1983, Nwadairo 1985, Rekhow 1987, Prinsloo \& Schoobee 1987, Staggis \& Otis 1996, Sharma \& Sinha 2000, Patgiri et al. 2001, Solanki et al. 2004, Boniamin laskar et al. 2005). However these studies did not investigate whether prevailing conditions were congenial for well being of fish. The merological approach for the studies on water bodies might not be useful in assessing suitability of water bodies for fish culture, whereas holological approach with emphasis on fish growth, water quality, and plankton diversity and density might be useful. Hence, there is a need to investigate variation in fish growth parameters along with physico-chemical and biological water quality parameters, to find out whether variation in growth and well being of fish are influenced by fluctuations in water quality. Studies on these lines are essential to assess suitability of water bodies for fish culture. In the present study we have compared growth co-efficient and relative condition factor of the major carp, Catla catla in two 
during different seasons in two perennial lakes to find out whether difference if any in water quality affects growth and relative condition factor.

\section{Materials and methods}

The present study was conducted on two perennial rain fed lakes viz. Yennehole lake (YL) (120 12' 22" $\mathrm{N}$ and $\left.760^{\circ} 31^{\prime} 12^{\prime \prime} \mathrm{E} 717 \mathrm{mts} \mathrm{MSL}\right)$ and Bilikere lake (BL) (12 $12^{\circ}$ $47^{\prime \prime} \mathrm{N}$ and $76^{\circ} 27^{\prime} 45^{\prime \prime} \mathrm{E} 810.46 \mathrm{mts}$ MSL). Both the lakes are being utilized for fish culture since $10-15$ years. YL is situated in southwest region of Mysore City and spread over about 200 acres and has depth of about $3-4$ mts. It is mainly used for culture of commercially important major carps. BL has an area of 32.5 acres and depth about 3 to $3.5 \mathrm{mts}$, which is utilized for irrigation, fish culture and domestic purposes.

The length - weight relationship of the major carp $C$. catla, was studied for two calendar years i.e. January 2002 to December 2003. During the study period length and weight of 15 specimen from each lake were recorded separately at monthly intervals. Later the data were pooled season wise (summer, monsoon and winter) to compute average for each season. The total length and the body weight were recorded immediately after the collection with the help of measuring board and weighing balance. The values of length and weight were determined to the nearest $\mathrm{mm}$ and $\mathrm{mg}$ respectively. The length- weight relationship was determined using the parabolic equation

( LeCren 1951), $\mathrm{W}=\mathrm{a} \mathrm{L}^{\mathrm{b}}$ (where $\mathrm{W}=$ weight of the fish, $\mathrm{a}=$ Multiplying constant, $\mathrm{b}=$ exponent of length, the growth factor and $\mathrm{L}=$ length of fish) for a given season. .

The correlation co-efficient ' $r$ 'was calculated by using following formula (Haynes, 1982).

\section{$\mathbf{r}=\Sigma \mathbf{X Y}-\mathbf{X} \Sigma \mathbf{Y} / \sqrt{ }\left[\left(\Sigma \mathbf{X}^{2}-\mathbf{X} \Sigma \mathbf{X}\right)\left(\Sigma \mathbf{Y}^{2}-\mathbf{Y} \Sigma \mathbf{Y}\right)\right]$}

The relative condition factor $(\mathrm{Kn})$ was computed by using formula (Le Cren, 1951),

$\mathrm{K}_{\mathrm{n}}=\mathrm{W}^{\mathrm{o}} / \mathrm{W}^{\mathrm{c}}$, Wherein $\mathrm{W}^{\mathrm{o}}=$ Observed weight of the fish, and $\mathrm{W}^{\mathrm{c}}=$ Calculated weight of the

fish.

Water samples were collected from three sampling sites at monthly intervals from both lakes. Samples were collected between 0800 to 10 00. Temperature, $\mathrm{pH}$ and DO were recorded on spot using portable kit. Total alkalinity, turbidity, TSS, BOD, hydrogen sulphide, phosphate nitrite and nitrate and ammonia were determined separately for all the samples in the laboratory by employing methods described in standard methods (APHA 1995). Arithmetic mean of each parameter computed considering values of three sampling sites. Monthly mean values were pooled to obtain season wise average.

Water samples for studying of phytoplankton and zooplankton diversity and density were collected simultaneously using nets of mesh size $20 \mu \mathrm{m}$ and $45 \mu \mathrm{m}$ respectively. One hundred liter of sub surface water was filtered through the nets and organisms were collected in glass bottles tied to the bottom of the net. Zooplankton were immediately transferred to specimen tubes with $4 \%$ formalin, whereas phytoplankton to Lugol's solution for later microscopic observation. Plankton counts were made in laboratory using Sedgewick rafter counting cells (Welch 1952). Values were computed and expressed in units/ liter. Phytoplankton were identified following keys of Desikachary 
(1959), Gonzalvis (1981) and Prescot,(1982). Zooplankton were identified following description of Edmondson (1959), Battish (1992), and Dhanapathi (2000).

\section{Results}

\section{Growth co-efficient and relative condition factor}

The growth co-efficient (b) was less than 3 in all the seasons in both the years in YL whereas in BL it was close to 3 or $>3$ in all seasons except monsoon, 2002. The correlation coefficient (r) ranged from 0.6004 (summer 2003) to 0.9251 (monsoon 2003) during different seasons of study period (two years) in YL whereas it ranged from 0.8376 (winter 2002) to 0.9248 (monsoon 2003) in BL. The values of correlation coefficient were statistically significant $(\mathrm{p}<0.05)$ for all the seasons and for both the lakes.

The relative condition factor $(\mathrm{Kn})$ was $<1$ in all the seasons in two years in YL whereas it was 1 or $>1$ in all the seasons during same period in BL.

Table 1. Length-weight relationship of Catla catla in Yennehole lake (YL) and Bilikere lake (BL) during 2002

\begin{tabular}{|c|c|c|c|c|c|c|}
\hline $\begin{array}{c}\text { Season \& } \\
\text { Lake }\end{array}$ & $\begin{array}{c}\text { Number } \\
\text { of Fish } \\
\text { studied }\end{array}$ & $\begin{array}{l}\text { Growth co- } \\
\text { efficient (b) }\end{array}$ & $\begin{array}{l}\text { Calculated } \\
\text { 'a' }\end{array}$ & $\mathbf{W}=\mathbf{a} \mathbf{L}^{\mathbf{b}}$ & $\begin{array}{c}\text { Correlation } \\
\text { co-efficient } \\
\text { (r) }\end{array}$ & $\begin{array}{c}\text { Relative } \\
\text { condition } \\
\text { factor } \\
(\mathrm{Kn})\end{array}$ \\
\hline YL & 60 & 2.61561 & 3.77477 & $3.7747 \mathrm{~L}^{2.61561}$ & 0.90014 & 0.87776 \\
\hline $\begin{array}{l}\text { Monsoon } \\
\text { BL }\end{array}$ & 60 & 2.6 & 0.12 & $0.12923 \mathrm{~L}^{2.60803}$ & 0.86 & 1.0084 \\
\hline YL & 60 & 2.10089 & 4.17332 & $4.17332 \mathrm{~L}^{2.10089}$ & 0.00452 & 0.9612 \\
\hline BL & 60 & 3.02897 & 0.93407 & $0.93407 \mathrm{~L}^{3.02897}$ & 0.8376 & 1.0539 \\
\hline YL & 60 & 2.71786 & 0.09595 & $0.09595 \mathrm{~L}^{2.71786}$ & 0.87046 & 0.93941 \\
\hline BL & 60 & 3.20223 & 0.01601 & $0.01601 L^{3.20223}$ & 0.85716 & 1.0350 \\
\hline
\end{tabular}

Table 2. Length-weight relationship of Catla catla in Yennehole lake (YL) and Bilikere lake (BL) during 2003

\begin{tabular}{|c|c|c|c|c|c|c|}
\hline $\begin{array}{c}\text { Season \& } \\
\text { Lake }\end{array}$ & $\begin{array}{l}\text { Number } \\
\text { of Fish } \\
\text { studied }\end{array}$ & $\begin{array}{l}\text { Growth co- } \\
\text { efficient (b) }\end{array}$ & $\begin{array}{l}\text { Calculated } \\
\text { 'a' }\end{array}$ & $\mathbf{W}=\mathbf{a L}^{\mathrm{b}}$ & $\begin{array}{c}\text { Correlation } \\
\text { co-efficient } \\
\text { (r) }\end{array}$ & $\begin{array}{c}\text { Relative } \\
\text { condition } \\
\text { factor } \\
(\mathbf{K n})\end{array}$ \\
\hline $\mathrm{YL}$ & 60 & 2.24267 & 0.45181 & $0.45181 \mathrm{~L}^{2.24267}$ & 0.92512 & 0.86503 \\
\hline $\begin{array}{l}\text { Monsoon } \\
\text { BL }\end{array}$ & 60 & 3.30474 & 0.01697 & $0.01697 \mathrm{~L}^{3.30474}$ & 0.9248 & 1.0177 \\
\hline YL & 60 & 2.09001 & 0.67138 & $0.67138 \mathrm{~L}^{2.09001}$ & 0.8742 & 0.98708 \\
\hline Winter & 60 & 3.34729 & 0.00932 & $0.00932 \mathrm{~L}^{3.34729}$ & 0.9012 & 1.0134 \\
\hline $\begin{array}{ll}\text { Summer } & \text { YL } \\
& \text { BL }\end{array}$ & 60 & 1.52285 & $\begin{array}{l}5.12968 \\
0.04285\end{array}$ & $\begin{array}{l}5.12968 \mathrm{~L}^{1.52285} \\
0.04285 \mathrm{~L}^{2.98760}\end{array}$ & $\begin{array}{r}0.60046 \\
0.87426\end{array}$ & $\begin{array}{l}0.8576 \\
1.0021\end{array}$ \\
\hline
\end{tabular}




\section{Physico-chemical water quality parameters}

Temperature in both lakes, in both the years of study did not show significant variation amongst different seasons. Further there was no significant variation in water temperature between both the lakes in all the seasons during the study period. The $\mathrm{pH}$ showed significant variation in both lakes in 2002, but not in 2003. The $\mathrm{pH}$ of BL was significantly lower compared to YL during winter of 2002 and monsoon and summer of 2003. During other seasons of the study period it did not differ significantly. Turbidity did not significantly vary amongst different seasons in YL in both years and in 2003 in BL. However, a significant variation was observed in 2002 in BL. Turbidity was significantly higher in YL compared to BL in all the seasons in both the years.

TSS did not significantly vary in BL in all seasons in both years whereas a seasonal variation was observed during 2003, but not in 2002 in YL. There was no significant difference between TSS of two lakes throughout study period except a significant decrease in BL in monsoon 2003 compared to YL. Total alkalinity did not significantly vary amongst different seasons in both years in YL. However it showed significant seasonal variation in 2002, but not in 2003 in BL. Total alkalinity of BL in winter and summer of both years was significantly lower compared to YL in both years, whereas it did not significantly differ during monsoon of both years.

DO content of YL did not show significant variation amongst different seasons in both years. DO content was significantly lower in winter and summer compared to monsoon in BL during 2002. A similar pattern was seen in 2003, however drop in winter 2003 was not significant compared to monsoon. In both years DO content of BL was significantly lower during winter and summer compared to that of YL whereas it did not differ during monsoon. The BOD did not significantly vary amongst different seasons in both years in YL whereas a significant seasonal variation was observed in 2003 in BL but not in 2002. Excepting monsoon in both years BOD of YL was significantly higher than BL in both years. Hydrogen sulphide content remained unchanged throughout the year in both lakes and it was significantly higher in YL compared to BL in all seasons. Nitrite concentration did not show significant seasonal variation in both years in BL, whereas it showed significant seasonal fluctuation in YL in 2003, but not in 2002. Nitrite concentration of BL was significantly lower in all the seasons compared to YL in both years. Nitrate concentration did not significantly vary amongst different seasons in both lakes in 2002. However in 2003 there was a significant seasonal variation in nitrate concentration in both lakes. The nitrate concentration of YL was significantly higher in all the seasons in both the years compared to BL. Phosphate concentration did not significantly vary amongst different seasons in both lakes in both years, and in all the seasons it was higher in YL compared to BL. In YL ammonia concentration varied significantly amongst different seasons in both years but in BL it did not. During entire study period ammonia concentration was significantly higher in YL compared to BL. 
Table 3. Seasonal variation in physico-chemical water quality parameters of Yennehole lake $(Y L)$ and Belikere lake (BL) during 2002

\begin{tabular}{|c|c|c|c|c|}
\hline & \multicolumn{5}{|c|}{ Yennehole Lake } \\
\hline Parameters & Monsoon & Winter & Summer & ANOVA F-value \\
\hline Temperature $\left({ }^{\circ} \mathrm{C}\right)$ & $26.05 \pm 0.71$ & $25.03 \pm 1.71$ & $26.37 \pm 0.81$ & $0.35 \mathrm{NS}$ \\
\hline $\mathrm{pH}$ & $9.01 \pm 0.11^{\mathrm{a}}$ & $9.45 \pm 0.13^{\mathrm{b}}$ & $9.02 \pm 0.009^{\mathrm{a}}$ & $5.476^{*}$ \\
\hline Turbidity $(\mathrm{NTU})$ & $54.34 \pm 8.07$ & $66.18 \pm 8.62$ & $76.24 \pm 8.67$ & $1.678 \mathrm{NS}$ \\
\hline TSS & $1.26 \pm 0.006$ & $0.67 \pm 0.53$ & $0.60 \pm 0.21$ & $3.293 \mathrm{NS}$ \\
\hline Total alkalinity & $960.37 \pm 60.4$ & $1080.4 \pm 135.7$ & $1165 \pm 110.4$ & $0.926 \mathrm{NS}$ \\
\hline $\mathrm{DO}$ & $18.56 \pm 2.87$ & $15.23 \pm 0.18$ & $17.36 \pm 3.57$ & $1.025 \mathrm{NS}$ \\
\hline $\mathrm{BOD}$ & $47.14 \pm 12.97$ & $24.71 \pm 1.81$ & $41.01 \pm 12.01$ & $1.277 \mathrm{NS}$ \\
\hline $\mathrm{H}_{2} \mathrm{~S}$ & $5.29 \pm 0.39$ & $2.58 \pm 023$ & $4.42 \pm 1.38$ & $2.698 \mathrm{NS}$ \\
\hline Nitrite & $0.70 \pm 0.17$ & $0.88 \pm 0.11$ & $0.47 \pm 0.11$ & $2.124 \mathrm{NS}$ \\
\hline Nitrate & $1.0 \pm 0.00$ & $0.94 \pm 0.1$ & $0.73 \pm 0.008$ & $2.544 \mathrm{NS}$ \\
\hline Phosphate & $1.87 \pm 0.18$ & $1.44 \pm 0.16$ & $1.55 \pm 0.1$ & $1.942 \mathrm{NS}$ \\
\hline Ammonia $(\mu \mathrm{g} / \mathrm{L})$ & $286.49 \pm 82.03^{\mathrm{a}}$ & $171.30 \pm 19.6^{\mathrm{b}}$ & $235.24 \pm 52.24^{\mathrm{a}}$ & $8.772 *$ \\
\hline & \multicolumn{5}{|c|}{ Bilikere Lake } & \\
\hline Temperature $\left({ }^{\circ} \mathrm{C}\right)$ & $26.81 \pm 1.43$ & $25.34 \pm 1.05$ & $26.81 \pm 1.43$ & $0.399 \mathrm{NS}$ \\
\hline $\mathrm{pH}$ & $9.09 \pm 0.009^{\mathrm{a}}$ & $8.52 \pm 0.14^{\mathrm{b}}$ & $8.99 \pm 0.009^{\mathrm{a}}$ & $24.961 *$ \\
\hline Turbidity $(\mathrm{NTU})$ & $30.98 \pm 3.09^{\mathrm{a}}$ & $17.28 \pm 6.34^{\mathrm{a}, \mathrm{b}}$ & $9.32 \pm 2.29^{\mathrm{b}}$ & $6.536 *$ \\
\hline TSS & $0.72 \pm 0.003$ & $0.45 \pm 0.34$ & $0.36 \pm 0.008$ & $2.823 \mathrm{NS}$ \\
\hline Total alkalinity & $899.16 \pm 15.62^{\mathrm{a}}$ & $426.6 \pm 67.16^{\mathrm{b}}$ & $859.99 \pm 57.84^{\mathrm{a}}$ & $6.391 *$ \\
\hline DO & $14.71 \pm 2.33^{\mathrm{a}}$ & $9.48 \pm 0.76^{\mathrm{b}}$ & $9.62 \pm 0.94^{\mathrm{b}}$ & $5.034 *$ \\
\hline BOD & $37.30 \pm 12.67$ & $14.43 \pm 1.70$ & $14.34 \pm 1.38$ & $3.172 \mathrm{NS}$ \\
\hline $\mathrm{H}_{2} \mathrm{~S}$ & $1.01 \pm 0.20$ & $0.56 \pm 0.1$ & $0.76 \pm 0.16$ & $1.994 \mathrm{NS}$ \\
\hline Nitrite & $0.004 \pm 0.003$ & $0.007 \pm 0.006$ & $0.0006 \pm 0$ & $0.606 \mathrm{NS}$ \\
\hline Nitrate & $0.49 \pm 0.16$ & $0.53 \pm 0.12$ & $0.39 \pm 0.12$ & $0.304 \mathrm{NS}$ \\
\hline Phosphate & $0.42 \pm 0.25$ & $0.17 \pm 0$ & $0.007 \pm 0$ & $1.311 \mathrm{NS}$ \\
\hline Ammonia $(\mu \mathrm{g} / \mathrm{L})$ & $39.24 \pm 8.04$ & $30.38 \pm 4.24$ & $28.16 \pm 12.08$ & $0.451 \mathrm{NS}$ \\
\hline & & \multicolumn{5}{|c|}{} \\
\hline
\end{tabular}

\begin{tabular}{|c|c|c|c|}
\hline Parameters & Monsoon & $\begin{array}{c}\text { Seasons } \\
\text { Winter }\end{array}$ & Summer \\
\hline Temperature & NS & NS & NS \\
\hline pH & NS & $\mathrm{P}<0.05$ & NS \\
\hline Turbidity & $\mathrm{P}<0.05$ & $\mathrm{P}<0.05$ & $\mathrm{P}<0.05$ \\
\hline TSS & NS & NS & NS \\
\hline Total alkalinity & NS & $\mathrm{P}<0.05$ & $\mathrm{P}<0.05$ \\
\hline DO & NS & $\mathrm{P}<0.05$ & $\mathrm{P}<0.05$ \\
\hline BOD & NS & $\mathrm{P}<0.05$ & $\mathrm{P}<0.05$ \\
\hline Hydrogen sulphide & $\mathrm{P}<0.05$ & $\mathrm{P}<0.05$ & $\mathrm{P}<0.05$ \\
\hline Nitrite & $\mathrm{P}<0.05$ & $\mathrm{P}<0.05$ & $\mathrm{P}<0.05$ \\
\hline Nitrate & $\mathrm{P}<0.05$ & $\mathrm{P}<0.05$ & $\mathrm{P}<0.05$ \\
\hline Phosphate & $\mathrm{P}<0.05$ & $\mathrm{P}<0.05$ & $\mathrm{P}<0.05$ \\
\hline Ammonia & $\mathrm{P}<0.05$ & $\mathrm{P}<0.05$ & $\mathrm{P}<0.05$ \\
\hline
\end{tabular}

Note: All parameters other than temp, $\mathrm{pH}$, turbidity and ammonia are $\mathrm{mg} / \mathrm{L}$. Mean values of each parameter compared by one way ANOVA followed by Duncan's multiple range test. Values with same superscript letter in different seasons for the given lake (rows) are not significantly different. Whereas those with different superscript letters are significantly $(\mathrm{P}<0.05)$ different. $* \mathrm{P}<0.05$.

Comparison of each parameter of YL and BL in each season as judged by Students t- test (significant if $\mathrm{P}<0.05)$ 
Table 4. Seasonal variation in physico-chemical water quality parameters of two lakes during 2003

\begin{tabular}{|c|c|c|c|c|}
\hline & \multicolumn{5}{|c|}{ Yennehole Lake } \\
\hline Parameters & Monsoon & Winter & Summer & ANOVA F-value \\
\hline Temperature $\left({ }^{\circ} \mathrm{C}\right)$ & $26.64 \pm 1.28$ & $25.49 \pm 1.72$ & $26.34 \pm 0.99$ & $0.158 \mathrm{NS}$ \\
\hline pH & $9.14 \pm 0.009$ & $9.29 \pm 0.009$ & $9.35 \pm 0.004$ & $2.481 \mathrm{NS}$ \\
\hline Turbidity $(\mathrm{NTU})$ & $76.33 \pm 9.0$ & $64.88 \pm 7.51$ & $50.77 \pm 14.77$ & $1.400 \mathrm{NS}$ \\
\hline TSS & $0.65 \pm 0.009^{\mathrm{a}}$ & $0.70 \pm 0.12^{\mathrm{a}}$ & $0.40 \pm 0.004^{\mathrm{b}}$ & $5.080 *$ \\
\hline Total alkalinity & $929.34 \pm 37.13$ & $955.97 \pm 38.17$ & $995.21 \pm 81.48$ & $0.348 \mathrm{NS}$ \\
\hline DO & $12.83 \pm 0.62$ & $15.0 \pm 0.96$ & $10.93 \pm 1.35$ & $3.947 \mathrm{NS}$ \\
\hline BOD & $48.67 \pm 8.46$ & $32.49 \pm 2.98$ & $44.59 \pm 7.11$ & $1.621 \mathrm{NS}$ \\
\hline $\mathrm{H}_{2} \mathrm{~S}$ & $3.56 \pm 0.99$ & $3.02 \pm 0.76$ & $3.49 \pm 0.72$ & $0.056 \mathrm{NS}$ \\
\hline Nitrite & $0.66 \pm 0.19^{\mathrm{a}}$ & $1.21 \pm 0.12^{\mathrm{b}}$ & $0.29 \pm 0.009^{\mathrm{a}}$ & $10.08^{*}$ \\
\hline Nitrate & $0.89 \pm 0.008^{\mathrm{a}, \mathrm{b}}$ & $1.19 \pm 0.13^{\mathrm{b}}$ & $0.63 \pm 0.008^{\mathrm{a}}$ & $7.432 *$ \\
\hline Phosphate & $1.72 \pm 0.22$ & $1.79 \pm 0.22$ & $1.62 \pm 0.007$ & $0.212 \mathrm{NS}$ \\
\hline Ammonia $(\mu \mathrm{g} / \mathrm{L})$ & $195.83 \pm 58.74^{\mathrm{c}}$ & $82.66 \pm 7.33^{\mathrm{a}}$ & $133.33 \pm 17.78^{\mathrm{b}}$ & $24.48^{*}$ \\
\hline & \multicolumn{5}{|c|}{ Bilikere Lake } & \\
\hline Temperature $\left({ }^{\circ} \mathrm{C}\right)$ & $27.02 \pm 1.29$ & $26.00 \pm 1.06$ & $27.37 \pm 0.34$ & $0.413 \mathrm{NS}$ \\
\hline pH & $8.98 \pm 0.008$ & $8.94 \pm 0.12$ & $8.88 \pm 0.21$ & $0.510 \mathrm{NS}$ \\
\hline Turbidity $(\mathrm{NTU})$ & $33.04 \pm 6.44$ & $20.97 \pm 6.61$ & $15.9 \pm 2.50$ & $2.540 \mathrm{NS}$ \\
\hline TSS & $0.37 \pm 0.004$ & $0.51 \pm 0.12$ & $0.32 \pm 0.007$ & $1.267 \mathrm{NS}$ \\
\hline Total alkalinity & $519.99 \pm 167.89$ & $415.83 \pm 74.44$ & $429.16 \pm 161.65$ & $0.161 \mathrm{NS}$ \\
\hline DO & $12.25 \pm 1.56^{\mathrm{b}}$ & $9.93 \pm 0.71^{\mathrm{a}, \mathrm{b}}$ & $7.24 \pm 0.62^{\mathrm{a}}$ & $5.652 *$ \\
\hline BOD & $37.99 \pm 8.81^{\mathrm{b}}$ & $16.30 \pm 1.88^{\mathrm{a}}$ & $17.54 \pm 3.20^{\mathrm{a}, \mathrm{b}}$ & $5.024 *$ \\
\hline $\mathrm{H}_{2} \mathrm{~S}$ & $0.70 \pm 0.17$ & $0.78 \pm 0.15$ & $0.62 \pm 0.10$ & $0.387 \mathrm{NS}$ \\
\hline Nitrite & $0.0008 \pm 0.0$ & $0.29 \pm 0.15$ & $0.0003 \pm 0.0$ & $3.544 \mathrm{NS}$ \\
\hline Nitrate & $0.24 \pm 0.10^{\mathrm{a}}$ & $0.71 \pm 0.11^{\mathrm{b}}$ & $0.31 \pm 0.005^{\mathrm{a}}$ & $7.098^{*}$ \\
\hline Phosphate & $0.50 \pm 0.41$ & $0.68 \pm 0.25$ & $0.21 \pm 0.16$ & $0.669 \mathrm{NS}$ \\
\hline Ammonia $(\mu \mathrm{g} / \mathrm{L})$ & $18.90 \pm 4.68$ & $16.46 \pm 5.10$ & $22.58 \pm 6.86$ & $0.300 \mathrm{NS}$ \\
\hline & & \multicolumn{4}{|c}{} \\
\hline
\end{tabular}

\begin{tabular}{|c|c|c|c|}
\hline \multirow{2}{*}{ Parameters } & \multicolumn{3}{|c|}{ Seasons } \\
\hline & Monsoon & Winter & Summer \\
\hline Temperature & NS & NS & NS \\
\hline $\mathrm{pH}$ & $P<0.05$ & NS & $\mathrm{P}<0.05$ \\
\hline Turbidity & $\mathrm{P}<0.05$ & $\mathrm{P}<0.05$ & $\mathrm{P}<0.05$ \\
\hline TSS & $\mathrm{P}<0.05$ & NS & NS \\
\hline Total alkalinity & NS & $\mathrm{P}<0.05$ & $\mathrm{P}<0.05$ \\
\hline DO & NS & $\mathrm{P}<0.05$ & $\mathrm{P}<0.05$ \\
\hline BOD & NS & $\mathrm{P}<0.05$ & $\mathrm{P}<0.05$ \\
\hline Hydrogen sulphide & $P<0.05$ & $\mathrm{P}<0.05$ & $\mathrm{P}<0.05$ \\
\hline Nitrite & $P<0.05$ & $\mathrm{P}<0.05$ & $\mathrm{P}<0.05$ \\
\hline Nitrate & $\mathrm{P}<0.05$ & $\mathrm{P}<0.05$ & $\mathrm{P}<0.05$ \\
\hline Phosphate & $P<0.05$ & $\mathrm{P}<0.05$ & $\mathrm{P}<0.05$ \\
\hline Ammonia & $\mathrm{P}<0.05$ & $\mathrm{P}<0.05$ & $\mathrm{P}<0.05$ \\
\hline
\end{tabular}

Note: All parameters other than temperature, $\mathrm{pH}$, turbidity and ammonia are $\mathrm{mg} / \mathrm{L}$. Mean values of each parameter compared by one way ANOVA followed by Duncan's multiple range test. Values with same superscript letter in different seasons for the given lake (rows) are significantly different. Whereas those with different superscript letters are significantly $(\mathrm{P}<0.05)$ different. $* \mathrm{P}<0.05$. Comparison of each parameter of $\mathrm{YL}$ and $\mathrm{BL}$ in each season as judged by Students t- test (significant if $\mathrm{P}<0.05$ ) 


\section{Diversity and density of plankton study}

Forty species of phytoplankton and 24 species of zooplankton were found in YL whereas 68 species of phytoplankton and 42 species of zooplankton were found in BL. Phytoplankton belonged to five different classes viz. cyanophyceae, chlorophyceae, bacillariophyceae, euglenophyceae and desmidiaceae. Zooplabnkton belonged to orders rotifera, copepoda, cladocera and ostracoda.

Phytoplankton population of YL was dominated by cyanophycean members in all the seasons of both years. Summer of 2002 recorded a significant increase in cyanophycean population compared to other two seasons. Same pattern was found in 2003 but the increase was not significant. Cyanophyceae in YL was represented by 10-12 species in different seasons of 2002 and 2003. Microcystis arruginosa kutz; was most dominating species among them and it was followed by several species belonging to genera, Oscillatoria, Anabaena and Nostoc. They formed a thin bloom on the water surface almost all the seasons of the study period. In BL cyanophycean density showed significant seasonal variation in 2003 but not in 2002. Cynophyte density in BL was significantly lower compared to YL in all the seasons of both the years. Chlorophyceae was third dominant group in YL and their density did not show significant seasonal variation in both the years. Chlorophytes were represented by 6-9 different species during study period. Phytoplankton of BL was dominated by chlorophytes, represented by 16-24 species during different seasons and their density showed significant seasonal variation in 2002, with an increase in density in summer. Similar pattern was found in 2003. Chlorophyceae density of BL did not show significant difference with that of YL in all the seasons in both years, except a significant decrease in monsoon 2002.

Bacillariophyceae were second dominant class in YL and their density did not show significant seasonal variation in both years. Bacillariophytes in YL were represented by 8-12 different species in different seasons of the study period. In BL bacillariophytes showed significant seasonal variation in both years. However, the pattern of variation was not similar in consecutive years. Density of bacillariophytes in YL and BL did not significantly differ in all the seasons, except a significant decrease in BL in monsoon 2002. Density of euglenophytes in YL did not show any significant seasonal variation in both the years and it was represented by 8-9 different species in different seasons of study period. In BL euglenophytes were represented by 6-7 species in different seasons of both the years and showed significant seasonal variation in their density in both years. However pattern of variation was not similar in consecutive years. Euglenophytes density of BL was lower than YL in all the seasons.

Desmids were conspicuous by their absence in YL in both the years whereas in BL desmids population was quite considerable in all the seasons of both the years and showed significant seasonal variation in both years, with high density in monsoon. They were represented by 8-11 species in different seasons of the study period.

Rotifer density in both lakes showed significant seasonal variation with high density in summer compared to other seasons. In all the seasons during two-year period rotifer density was significantly lower in BL compared to YL. However, rotifers were represented by more number of species in BL than YL. Copepoda in YL were represented by 3-4 species their density showed significant seasonal fluctuation in 2002, but not in 2003. In BL copepod density was significantly increased in winter 2002.

Copepodes were represented by 5-7 species in different seasons in BL and their density in BL during all the seasons of 2002 and in winter of 2003 was significantly lower compared to YL. 
Table 5. Seasonal variation in plankton density in Yennehole lake and Bilikere lake, 2002

\begin{tabular}{|c|c|c|c|c|c|}
\hline $\begin{array}{c}\text { Plankton } \\
\text { Lake }\end{array}$ & & Monsoon & Winter & Summer & $\begin{array}{c}\text { ANOVA } \\
\text { F-value }\end{array}$ \\
\hline \multirow[t]{2}{*}{ Cyanophyceae } & YL & $\begin{array}{c}29050 \pm 4031.23^{\mathrm{a}} \\
(12)\end{array}$ & $\begin{array}{c}34025 \pm 4219.86^{\mathrm{a}, \mathrm{b}} \\
(12)\end{array}$ & $\begin{array}{c}40870 \pm 1265.66^{\mathrm{b}} \\
(10)\end{array}$ & $\begin{array}{c}5.963 \\
(\mathrm{P}<0.05)\end{array}$ \\
\hline & BL & $\begin{array}{c}6425 \pm 782.49 \\
(09)\end{array}$ & $\begin{array}{c}9300 \pm 1149.63 \\
(07)\end{array}$ & $\begin{array}{c}9750 \pm 1354.31 \\
(08)\end{array}$ & $\begin{array}{l}2.591 \\
(\mathrm{NS})\end{array}$ \\
\hline \multirow[t]{2}{*}{ Chlorophyceae } & YL & $\begin{array}{c}9500 \pm 1666.33 \\
(09)\end{array}$ & $\begin{array}{c}8000 \pm 804.15 \\
(08)\end{array}$ & $\begin{array}{c}9250 \pm 717.05 \\
(08)\end{array}$ & $\begin{array}{l}0.492 \\
(\mathrm{NS})\end{array}$ \\
\hline & $\mathrm{BL}$ & $\begin{array}{c}6725 \pm 601.90^{\mathrm{a}} \\
(13)\end{array}$ & $\begin{array}{c}9800 \pm 963.50^{\mathrm{a}, \mathrm{b}} \\
(16)\end{array}$ & $\begin{array}{c}12100 \pm 1948.07^{\mathrm{b}} \\
(16)\end{array}$ & $\begin{array}{c}5.290 \\
(\mathrm{P}<0.05)\end{array}$ \\
\hline \multicolumn{2}{|c|}{ Bacillariophyceae } & $\begin{array}{c}10600 \pm 1292.92 \\
(09)\end{array}$ & $\begin{array}{c}9050 \pm 494.13 \\
(11)\end{array}$ & $\begin{array}{c}9300 \pm 1068.48 \\
(12)\end{array}$ & $\begin{array}{l}0.679 \\
(\mathrm{NS})\end{array}$ \\
\hline & BL & $\begin{array}{c}6200 \pm 302.76^{\mathrm{a}} \\
(10) \\
\end{array}$ & $\begin{array}{c}11200 \pm 721.11^{\mathrm{b}} \\
(11)\end{array}$ & $\begin{array}{c}8740 \pm 186.84^{\mathrm{a}, \mathrm{b}} \\
(10)\end{array}$ & $\begin{array}{c}6.018 \\
(\mathrm{P}<0.05)\end{array}$ \\
\hline \multirow[t]{2}{*}{ Euglenophyceae } & YL & $\begin{array}{c}9400 \pm 355.90 \\
(08)\end{array}$ & $\begin{array}{c}10600 \pm 948.68 \\
(09)\end{array}$ & $\begin{array}{c}8200 \pm 989.10 \\
(08)\end{array}$ & $\begin{array}{l}2.155 \\
(\mathrm{NS})\end{array}$ \\
\hline & BL & $\begin{array}{c}7175 \pm 661.28^{\mathrm{b}} \\
(06)\end{array}$ & $\begin{array}{c}4200 \pm 452.76^{\mathrm{a}, \mathrm{b}} \\
(07)\end{array}$ & $\begin{array}{c}3550 \pm 545.43^{\mathrm{a}} \\
(07)\end{array}$ & $\begin{array}{c}11.925 \\
(\mathrm{P}<0.05)\end{array}$ \\
\hline \multirow{2}{*}{ Desmids } & YL & NIL & NIL & NIL & NIL \\
\hline & $\mathrm{BL}$ & $7900 \pm 511.53^{b}$ & $3800 \pm 374.16^{\mathrm{a}}$ & $4500 \pm 254.95^{\mathrm{a}}$ & $\begin{array}{c}30.921 \\
(\mathrm{P}<0.05)\end{array}$ \\
\hline \multirow[t]{2}{*}{ Rotifers } & YL & $\begin{array}{c}643 \pm 32.54^{\mathrm{a}} \\
(10)\end{array}$ & $\begin{array}{c}613 \pm 36.77^{\mathrm{a}} \\
(12)\end{array}$ & $\begin{array}{c}858 \pm 86.43^{b} \\
\text { (11) }\end{array}$ & $\begin{array}{c}5.421 \\
(\mathrm{P}<0.05)\end{array}$ \\
\hline & BL & $\begin{array}{c}218 \pm 26.77^{\mathrm{a}} \\
(17)\end{array}$ & $\begin{array}{c}138 \pm 15.87^{\mathrm{a}} \\
(15)\end{array}$ & $\begin{array}{c}422 \pm 68.43^{b} \\
\text { (19) }\end{array}$ & $\begin{array}{c}11.382 \\
(\mathrm{P}<0.05)\end{array}$ \\
\hline \multirow[t]{2}{*}{ Copepods } & YL & $\begin{array}{c}580 \pm 67.56^{\mathrm{a}} \\
(04)\end{array}$ & $\begin{array}{c}636 \pm 67.42^{a} \\
(04)\end{array}$ & $\begin{array}{c}298 \pm 14.86^{\mathrm{b}} \\
(04)\end{array}$ & $\begin{array}{c}13.034 \\
(\mathrm{P}<0.05)\end{array}$ \\
\hline & $\mathrm{BL}$ & $\begin{array}{c}171 \pm 32.60^{\mathrm{a}} \\
(07)\end{array}$ & $\begin{array}{c}292 \pm 7.39^{b} \\
(06)\end{array}$ & $\begin{array}{c}168 \pm 12.49^{\mathrm{a}} \\
(07)\end{array}$ & $\begin{array}{c}12.999 \\
(\mathrm{P}<0.05)\end{array}$ \\
\hline \multirow[t]{2}{*}{ Cladocera } & $\mathrm{YL}$ & $\begin{array}{c}182 \pm 16.87 \\
(04)\end{array}$ & $\begin{array}{c}210 \pm 11.80 \\
(04)\end{array}$ & $\begin{array}{c}146 \pm 12.86 \\
(03)\end{array}$ & $\begin{array}{l}2.641 \\
(\mathrm{NS})\end{array}$ \\
\hline & BL & $\begin{array}{c}122 \pm 20.89^{\mathrm{a}} \\
(05)\end{array}$ & $\begin{array}{c}248 \pm 23.62^{b} \\
(05)\end{array}$ & $\begin{array}{c}108 \pm 20.86^{\mathrm{a}} \\
(06)\end{array}$ & $\begin{array}{c}12.496 \\
(\mathrm{P}<0.05)\end{array}$ \\
\hline \multirow[t]{2}{*}{ Ostracods } & YL & $\begin{array}{c}74 \pm 12.36^{\mathrm{a}} \\
(04)\end{array}$ & $\begin{array}{c}77 \pm 18.96^{\mathrm{a}} \\
(03)\end{array}$ & $\begin{array}{c}38 \pm 2.86^{b} \\
(03)\end{array}$ & $\begin{array}{c}5.665 \\
(\mathrm{P}<0.05)\end{array}$ \\
\hline & BL & $\begin{array}{c}57 \pm 11.56^{\mathrm{a}} \\
(06)\end{array}$ & $\begin{array}{c}80 \pm 12.94^{b} \\
(06)\end{array}$ & $\begin{array}{c}56 \pm 3.16^{\mathrm{a}} \\
(04)\end{array}$ & $\begin{array}{c}11.600 \\
(\mathrm{P}<0.05)\end{array}$ \\
\hline
\end{tabular}

\begin{tabular}{|c|c|c|c|}
\hline \multirow{2}{*}{ Plankton } & \multicolumn{3}{|c|}{ Seasons } \\
\cline { 2 - 4 } & Monsoon & Winter & Summer \\
\hline Cyanophyceae & $\mathrm{P}<0.05$ & $\mathrm{P}<0.05$ & $\mathrm{P}<0.05$ \\
\hline Chlorophyceae & $\mathrm{P}<0.05$ & $\mathrm{NS}$ & $\mathrm{NS}$ \\
\hline Bacillariophyceae & $\mathrm{P}<0.05$ & $\mathrm{NS}$ & $\mathrm{NS}$ \\
\hline Euglenophyceae & $\mathrm{NS}$ & $\mathrm{P}<0.05$ & $\mathrm{~N}$ \\
\hline Desmids & $\mathrm{il}$ & $\mathrm{Nil}$ & $\mathrm{P}<0.05$ \\
\hline Rotifers & $\mathrm{P}<0.05$ & $\mathrm{P}<0.05$ & $\mathrm{P}<0.05$ \\
\hline Copepods & $\mathrm{P}<0.05$ & $\mathrm{P}<0.05$ & $\mathrm{NS}$ \\
\hline Cladocera & $\mathrm{NS}$ & $\mathrm{NS}$ & $\mathrm{NS}$ \\
\hline Ostracods & $\mathrm{NS}$ & $\mathrm{NS}$ & \\
\hline
\end{tabular}


Table 6. Seasonal variation in plankton density in Yennehole lake and Bilikere lake, 2003

\begin{tabular}{|c|c|c|c|c|c|}
\hline $\begin{array}{c}\text { Plankton } \\
\text { (units/lits) }\end{array}$ & & Monsoon & Winter & Summer & $\begin{array}{c}\text { ANOVA } \\
\text { F-value }\end{array}$ \\
\hline \multirow[b]{2}{*}{ Cyanophyceae } & YL & $\begin{array}{c}31875 \pm 4248.79 \\
\text { (13) }\end{array}$ & $\begin{array}{c}34475 \pm 4819.98 \\
(12)\end{array}$ & $\begin{array}{c}45100 \pm 6258.06 \\
(10)\end{array}$ & $\begin{array}{l}2.062 \\
\text { (NS) }\end{array}$ \\
\hline & BL & $\begin{array}{c}8100 \pm 866.02^{\mathrm{a}} \\
(11)\end{array}$ & $\begin{array}{c}14250 \pm 1374.46^{\mathrm{b}} \\
(09)\end{array}$ & $\begin{array}{c}11100 \pm 1707.82^{\mathrm{a}, \mathrm{b}} \\
(11)\end{array}$ & $\begin{array}{c}5.107 \\
(\mathrm{P}<0.05)\end{array}$ \\
\hline \multirow{2}{*}{ Chlorophyceae } & YL & $\begin{array}{c}9800 \pm 904.23 \\
(06)\end{array}$ & $\begin{array}{c}9500 \pm 665.83 \\
(09)\end{array}$ & $\begin{array}{c}10100 \pm 854.40 \\
(08)\end{array}$ & $\begin{array}{l}0.136 \\
\text { (NS) }\end{array}$ \\
\hline & BL & $\begin{array}{c}9100 \pm 782.09^{\mathrm{a}} \\
(16)\end{array}$ & $\begin{array}{c}7500 \pm 823.60^{\mathrm{a}, \mathrm{b}} \\
(16)\end{array}$ & $\begin{array}{c}11550 \pm 1060.26^{b} \\
(16)\end{array}$ & $\begin{array}{c}5.171 \\
(\mathrm{P}<0.05)\end{array}$ \\
\hline \multirow{2}{*}{ Bacillariophycea } & YL & $\begin{array}{c}8000 \pm 580.22 \\
(09)\end{array}$ & $\begin{array}{c}10550 \pm 1447.12 \\
(10)\end{array}$ & $\begin{array}{c}9125 \pm 604.66 \\
(08)\end{array}$ & $\begin{array}{l}1.752 \\
\text { (NS) }\end{array}$ \\
\hline & $\begin{array}{l}\mathrm{ae} \\
\mathrm{BL}\end{array}$ & $\begin{array}{c}7900 \pm 491.59^{\mathrm{a}} \\
(10)\end{array}$ & $\begin{array}{c}10600 \pm 960.03^{\mathrm{b}} \\
(12)\end{array}$ & $\begin{array}{c}13800 \pm 875.59^{c} \\
(13)\end{array}$ & $\begin{array}{c}13.119 \\
(\mathrm{P}<0.05)\end{array}$ \\
\hline \multirow{2}{*}{ Euglenophyceae } & YL & $\begin{array}{c}8100 \pm 1191.63 \\
(08)\end{array}$ & $\begin{array}{c}8050 \pm 375.27 \\
(09)\end{array}$ & $\begin{array}{c}10425 \pm 1326.88 \\
(08)\end{array}$ & $\begin{array}{l}1.663 \\
\text { (NS) }\end{array}$ \\
\hline & BL & $\begin{array}{c}6700 \pm 467.26^{\mathrm{a}} \\
(07)\end{array}$ & $\begin{array}{c}2850 \pm 370.80^{\mathrm{b}} \\
(06)\end{array}$ & $\begin{array}{c}5325 \pm 525.0^{\mathrm{a}} \\
(07)\end{array}$ & $\begin{array}{c}18.064 \\
(\mathrm{P}<0.05)\end{array}$ \\
\hline \multirow[b]{2}{*}{ Desmids } & YL & NIL & NIL & NIL & NIL \\
\hline & $\mathrm{BL}$ & $\begin{array}{c}7425 \pm 306.52^{\mathrm{c}} \\
\text { (09) }\end{array}$ & $\begin{array}{c}4650 \pm 233.63^{\mathrm{b}} \\
(09)\end{array}$ & $\begin{array}{c}3600 \pm 285.77^{\mathrm{a}} \\
(08)\end{array}$ & $\begin{array}{c}50.897 \\
(\mathrm{P}<0.05)\end{array}$ \\
\hline \multirow[t]{2}{*}{ Rotifers } & YL & $\begin{array}{l}495 \pm 56.93^{\mathrm{a}} \\
(12)\end{array}$ & $\begin{array}{l}561 \pm 70.28^{\mathrm{a}} \\
(12)\end{array}$ & $\begin{array}{c}947 \pm 145.98^{\mathrm{b}} \\
(10)\end{array}$ & $\begin{array}{c}6.087 \\
(\mathrm{P}<0.05)\end{array}$ \\
\hline & BL & $\begin{array}{c}172 \pm 24.98^{\mathrm{a}} \\
(18)\end{array}$ & $\begin{array}{c}228 \pm 32.01^{\mathrm{a}} \\
\quad(17)\end{array}$ & $\begin{array}{c}354 \pm 28.69^{\mathrm{b}} \\
(20)\end{array}$ & $\begin{array}{c}10.315 \\
(\mathrm{P}<0.05)\end{array}$ \\
\hline \multirow[b]{2}{*}{ Copepods } & $\mathrm{YL}$ & $\begin{array}{c}324 \pm 67.23^{\mathrm{a}} \\
(03)\end{array}$ & $\begin{array}{c}810 \pm 130.36^{\mathrm{b}} \\
(04)\end{array}$ & $\begin{array}{c}270 \pm 13.39^{\mathrm{a}} \\
(04)\end{array}$ & $\begin{array}{c}12.232 \\
(\mathrm{P}<0.05)\end{array}$ \\
\hline & BL & $\begin{array}{c}236 \pm 57.89 \\
(07)\end{array}$ & $\begin{array}{c}331 \pm 30.88 \\
(06)\end{array}$ & $\begin{array}{c}198 \pm 38.75 \\
(05)\end{array}$ & $\begin{array}{l}2.499 \\
\text { (NS) }\end{array}$ \\
\hline \multirow[b]{2}{*}{ Cladocera } & YL & $\begin{array}{c}172 \pm 32.12^{\mathrm{a}} \\
(04)\end{array}$ & $\begin{array}{c}168 \pm 16.46^{\mathrm{a}} \\
(03)\end{array}$ & $\begin{array}{c}116 \pm 12.86^{\mathrm{a}} \\
(04)\end{array}$ & $\begin{array}{c}6.092 \\
(\mathrm{P}<0.05)\end{array}$ \\
\hline & BL & $\begin{array}{c}125 \pm 20.69^{\mathrm{a}} \\
(05)\end{array}$ & $\begin{array}{c}164 \pm 23.20^{\mathrm{a}} \\
(07)\end{array}$ & $\begin{array}{c}224 \pm 18.38^{\mathrm{b}} \\
(07)\end{array}$ & $\begin{array}{c}9.326 \\
(\mathrm{P}<0.05)\end{array}$ \\
\hline \multirow{2}{*}{ Ostracods } & $\mathrm{YL}$ & $\begin{array}{c}38 \pm 6.42 \\
(04)\end{array}$ & $\begin{array}{c}46 \pm 8.02 \\
(04)\end{array}$ & $\begin{array}{c}28 \pm 2.06 \\
(03)\end{array}$ & $\begin{array}{l}1.891 \\
\text { (NS) }\end{array}$ \\
\hline & $\mathrm{BL}$ & $\begin{array}{c}10 \pm 3.5^{\mathrm{b}} \\
(04)\end{array}$ & $\begin{array}{c}46 \pm 4.08^{\mathrm{a}} \\
(06)\end{array}$ & $\begin{array}{c}58 \pm 8.75^{\mathrm{a}} \\
(04)\end{array}$ & $\begin{array}{c}19.358 \\
(\mathrm{P}<0.05)\end{array}$ \\
\hline
\end{tabular}

\begin{tabular}{|c|c|c|c|}
\hline \multirow{2}{*}{ Plankton } & \multicolumn{3}{|c|}{ Seasons } \\
\cline { 2 - 4 } & Monsoon & Winter & Summer \\
\hline Cyanophyceae & $\mathrm{P}<0.05$ & $\mathrm{P}<0.05$ & $\mathrm{P}<0.05$ \\
\hline Chlorophyceae & $\mathrm{NS}$ & $\mathrm{NS}$ & $\mathrm{NS}$ \\
\hline Bacillariophyceae & $\mathrm{NS}$ & $\mathrm{NS}$ & $\mathrm{NS}$ \\
\hline Euglenophyceae & $\mathrm{NS}$ & $\mathrm{P}<0.05$ & $\mathrm{~N}$ \\
\hline Desmids & $\mathrm{Nil}$ & $\mathrm{Nil}$ & $\mathrm{P}<0.05$ \\
\hline Rotifers & $\mathrm{P}<0.05$ & $\mathrm{P}<0.05$ & $\mathrm{P}<0.05$ \\
\hline Copepods & $\mathrm{NS}$ & $\mathrm{P}<0.05$ & $\mathrm{NS}$ \\
\hline Cladocera & $\mathrm{NS}$ & $\mathrm{NS}$ & $\mathrm{NS}$ \\
\hline Ostracods & $\mathrm{P}<0.05$ & & \\
\hline
\end{tabular}


Cladoceran density did not show significant seasonal variation in YL in both the years whereas they showed significant variation in their density in BL in both years. There was no significant difference between cladodoceran population of YL and BL in all the seasons of the study period.

The seasonal variation in density of ostracods in 2002 was significant in both lakes whereas in 2003, it was significant only in BL but not in YL.

\section{Discussion}

In the present study seasonal variation in different physico-chemical parameters, which are known to influence well being of fish and growth of plankton have been studied, to find out whether differences in these parameters in two lakes is accompanied by difference in growth co- efficient and relative condition factor of a major carp $C$. catla.

In the present study water temperature of $\mathrm{YL}$ ranged from $25.03^{\circ} \mathrm{C}$ to $26.64^{\circ} \mathrm{C}$ in both the years where as that of BL was $25.34^{\circ} \mathrm{C}$ to $27.37^{\circ} \mathrm{C}$. Since a range of $28-32^{\circ} \mathrm{C}$ in tropical waters (IFAS: Insttitute of food and agricultural sciences, University of Florida,Circular-1051, Jinghran ,1968) is congenial for optimal growth of fish, both the lakes under study showed temperature closer to the lower limits of the optimum range. Similarly, the DO content of both the lakes during entire study period was conducive for fish growth as it was well above the minimum required amount (i.e. $5 \mathrm{mg} / \mathrm{lit}$., Alabaster \& Lloid, 1980). Since higher levels of total suspended solids clog the fish gills, their concentration less than $25 \mathrm{mg} /$ lit is preferred (Maitland, 1990). In our study TSS level was well within the range in both lakes. However, other physico-chemical parameters showed significant difference between two lakes and some of them were in undesirable level in YL compared to BL. For instance, low turbidity (20-30 NTU) is desirable for fish culture (Zweig, 1989) as high level turbidity affects the photosynthetic process and there by the potential yield of the lake (Sukumaran \& Das 2005). In BL turbidity level was within the desirable range (9.3 NTU - 33.04 NTU) whereas that in YL was (50.77NTU - 76.33NTU) higher than desired range. Similarly higher alkalinity $(\mathrm{pH}>9)$ in water bodies is unsuitable for good fish production (Boyd 1979). The water $\mathrm{pH}$ in YL was always higher than 9 whereas in BL it was less than 9 in majority of the seasons. Likewise total alkalinity in YL (929.34 mg/lit to $1016.5 \mathrm{mg} / \mathrm{lit})$ was remarkably higher than optimal range (100 to $400 \mathrm{mg} / \mathrm{lit}$, Schroeder, 1980) for fish culture. Whereas in BL, excepting monsoon it was within desired range of alkalinity preferred for fish culture.

BOD indicates the presence of organic load in a water body and waters having BOD more than 35 to $45 \mathrm{mg} / \mathrm{lit}$ are not good for fish culture (Pande \& Sharma 1999). In the present study BOD level in YL exceeded the preferred range in all the seasons except in winter in contrast to BL wherein it was in preferred range in all seasons except monsoon.

Phosphate is a nutrient which causes rich phytoplankton crop (Moss, 1993). An optimum level 0.1 to $0.2 \mathrm{mg} / \mathrm{lit}$ phosphate (Sreenivasan, 1965) is needed for growth of plankton. In our study, in YL the phosphate concentration was several folds higher than optimal level (0.1 to $0.2 \mathrm{mg} / \mathrm{lit}$, Sreenivasan, 1965) needed to support phytoplankton growth. Whereas in BL it was within desirable range except in monsoon.

Nitrite could be hazardous to fish if it exceeds the permissible range (Train \& Russel 1979) which is $0.015 \mathrm{mg} / \mathrm{lit}$ for salmonids (Iwama et al. 2000) and generally $0.1 \mathrm{mg} / \mathrm{lit}$ considered tolerable range in tropics (Hart \& Reynolds, 2002). In the present study YL 
exceeded the tolerance limit whereas in BL it was very well with in the desirable range throughout the year. Minimum level of nitrate required for the lake to be productive is $0.1 \mathrm{mg} /$ lit (Srinivasan 1965, Hart \& Reynolds, 2002). In the present study nitrate content although exceeded the optimal level in both the lakes, the concentration of nitrate was far higher in YL than BL. The excessive level of nutrients in YL was reflected in the presence of algal bloom during most part of the study period.

Unionized ammonia in the range of $0.02-0.2 \mathrm{mg} / \mathrm{lit}$ is toxic (Alabaster \& Lloyd, 1980., Joseph et al. 1993) to fishes as excessive ammonia in water tends to block $\mathrm{O}_{2}$ transfer from gills to the blood (Smart, 1978). In the present study ammonia content in YL was not only significantly higher than BL but was also in toxic level.

Hydrogen sulphide in water bodies is another indication of pollution (Oslen \& Sommerfeld 1977). In the present study $\mathrm{H}_{2} \mathrm{~S}$ is the only parameter, which was in undesirable range in both the lakes. It ranged from $2.58 \mathrm{mg} / \mathrm{lit}$ to $5.29 \mathrm{mg} / \mathrm{lit}$ in YL and from $0.56 \mathrm{mg} /$ lit to $1.01 \mathrm{mg} / \mathrm{lit}$ in $\mathrm{BL}$. High levels of $\mathrm{pH}$, total alkalinity, turbidity, BOD, H2S, phosphate and nitrite were reported in number of studies in different lakes in India and outside, to cite a few, Hutchinson 1957, Verma 1967, Banergia 1967, Saxena \& Adoni 1973, Ayyappan \& Gupta 1981, Yousuf et al. 1986, Kaur et al. 2000, Ragavendra \& Hosmani 2002. However these studies did not focus on the fact that whether these conditions interfered with growth and well being of fish in these water bodies. The isometric growth of fish under optimum conditions follows length-weight relationship, wherein weight is cube of length.( cube rule , Le Cren, 1951). In the length weight relationship equation $\left(\mathrm{W}=\mathrm{aL}^{\mathrm{b}}\right), \mathrm{b}$ is the growth co-efficient and its value is 3 (Allen, 1938) under optimal conditions. Hile (1936) and Martin (1949) opined that value of $b$ usually lies between 2.5 and 4 . Hence, in the study of length weight relationship, value of b because less than 2.5 can be considered as subnormal growth of fish in that given lake. Further the relative condition factor $(\mathrm{Kn})$ is an expression used to asses the condition of fish, and $\mathrm{Kn}$ value 1 or more than 1 is considered as well being of fish. The present study, which compares these parameters of $C$. catla in two lakes, for two calendar years, reveals a few interesting facts. The growth coefficient (b) was 3 or close to 3 in majority of seasons, accompanied by Kn value 1 are more than 1 in BL, where as it was less than 3 accompanied by Kn value less than 1 in all the seasons in YL. These observations clearly indicate better growth and health (well being) of $C$. catla in BL than in YL. Although several earlier studies on fish growth reveled sub optimal growth of fish they did not provide evidence of any causative factor. In the present study, the sub optimal growth of $C$. catla in YL was accompanied by high $\mathrm{pH}$, turbidity, total alkalinity, BOD, nitrate, nitrite, phosphate, hydrogen sulphide and ammonia which were beyond normal range for fish culture in contrast to lower values of these parameters accompanied by normal growth of $C$. catla in BL. Higher levels of these physico-chemical factors directly or indirectly interfere with fish physiology and affect their growth. For instance high turbidity (Zweig, 1989) reduces photosynthetic zone resulting in night time decline of DO and higher $\mathrm{pH}$ (Boyd, 1979) influences the blood $\mathrm{pH}$ and causes alkalosis; damages skin, gills and eyes; and increases mucus production. Similarly, oxygen consumption of fish is affected by high nitrite, nitrate and ammonia (Tilak et al. 2005) as nitrate in addition high ammonia interferes with oxygen transport from gills to blood ( Smart, 1978.,Lewis \& Morris, 1986., Datta et al. 2005) and damages gills. Sub-optimal levels of unionized ammonia $(0.1-0.42 \mathrm{mg} / \mathrm{lit})$ causes significant variation in condition factor (Datta et al. 2005). Likewise higher than tolerable level of hydrogendulphide might cause death to the fishes or at the very least 
stress (Barthelmes \& Bramick 2003). The combined effect of all these physico-chemical factors might induce stress response as suggested by Iwama (2000). It is well known that stress adversely affects growth of animals. Hence, the sub optimal growth of $C$. catla as indicated by deviation from cube rule and lower Kn values in YL are due to prevailing physico-chemical conditions in YL. This view is further supported by the fact that the above parameters which are in normal range in BL in which not only growth co-efficient of C.catla obeyed the cube rule but also Kn values indicated well being of fish.

The difference in physico-chemical characteristic in two study lakes was also reflected in plankton density and diversity; indicating water contamination in YL. Although phytoplankton being producers play a key role in aquatic food chain, higher nutrient levels cause their bloom, which will be detrimental to fish by various effects. Nutrient enrichment resulting in algal bloom is indicated by excessive growth of certain algal s genera ; Microcystis , Anabaena, Oscillatoria, Scenedesmus, Pediastrum, Fragellaria etc. (Palmer, 1980., Bush \& Welch, 1972). In the present study YL showed algal bloom throughout the year and was dominated by cyanophytes, especially Microcysties , Oscilotoria, Anabina etc and bacillariophytes such as Navicula, Nitzschia, Synedra etc, which indicate nutrient load and sewage pollution (Palmer,1980). This view is also supported by the presence high density of rotifers viz. Brachionus angularis, B. quadricormis, Keratella cochlearis, Felinia longiseta, Polyarthra vulgaris and Conochilus dassaurius, which are also an indicator of high nutrient load (Sharma et al. 1999., Bahura et al. 1993). In contrast in BL, low nutrient levels compared to YL was accompanied by the presence of desmids, high density of chlorophyceae which grow better in waters with low organic matter and high DO (Goldman \& Home, 1983). Cyanophytes dominated by M. aeruginosa are found to produce two toxins viz; hepatotoxin, microcystin and a neurotoxin, anatoxin and adversely effect the well being of the fish (Ballot et al. 2003). Cyanophycean bloom also causes "off flavor" either by producing a substance called MIB (Methyl isoborneal) or by the decomposition process of their own counterparts (Martin, et al; 1994). In our study we observed bloom and off flavor in YL quite often . In addition, plankton diversity was more in BL than in YL. Put together these biological parameters indicate better conditions in BL than in YL. Hence the study by comparing growth

co-efficient and relative condition factor of same species in two water bodies which differ in physico-chemical properties, first time provides an evidence for the fact that water quality parameters in undesirable range in natural water bodies interfere with growth and well being of fish. It is to be noted that though the conditions in YL were never severe enough to cause fish deaths, they interfered with growth and well being of C.catla. Hence such studies will be useful in assessing the suitability of ponds for fish culture.

Acknowledgements. First author is grateful to University Grants Commission, India, for the award of teacher fellowship under the faculty improvement program. 


\section{REFERENCES}

[1] Alabaster, J.S. \& Lloyd, R. (1980): Water quality criteria for fresh water fish. Butterworths, London.

[2] Allen, K.R. (1938): Some observation on the biology of the trout (Salmotrutta) in windermere. - Journal of Animal Ecology. 7:333-349.

[3] APHA. (1995): Standard methods for the examination of water and wastewater. American Public health association, $19^{\text {th }}$ Edition, Washington D.C.

[4] Ayyappan, S. \& Gupta, T.R. (1981): Limnology of Ramasamudra tank-Hydrograph, Mysore. - J.Agric. Sc., 15: 305-312.

[5] Bahura, C.K., Bahura, P., Saxena, M.M. (1993): Zooplankton community of shivbari temple tank, Bikaner. - Journal of Ecology. 5: 5-8.

[6] Ballot, A., Pflugmacher, S., Wiegand, C., Kotut , K. Krienitz, K. (2003): Cyanobacterial toxins in Lake Baringo, Kenya. - Limnologica 33:2-9.

[7] Banerjea,S.M. (1967): Water quality and soil condition of fish ponds in some states of India in relation to fish production. - Indian J. Fish. 14(1\&2): 115-144.

[8] Barthelmes, D. \& Bramick, U. (2003): Variability of Cyprinid lake ecosystem with special emphasis on the native fish fauna under intensive fisheries management including common carp (Cyprinus carpio) and Silver carp (Hypophthalmichthys molitrix). - SO Limnologica, 72:331-342.

[9] Bashirulla, A.K.M \& Kader, M.A. (1970): The Length-Weight relationship \& condition of Trichiurus savala cuv and val. Pakistan Journal of Science. - Ind. Res 13 (4): 414-419.

[10] Battish, S.K. (1992): Freshwater zooplankton of India. - Oxford \& IBH publishing co. Pvt. Ltd.

[11] Boni Amin Laskar, Aren Pujen \& Pronabnath. (2005): Study of the Length-Weight relationship \& population structure of Chocolate Mahaseer Neolissocheilus Hexagonolepis in Shelly lake of Arunachalpradesh. - Indian Journal of Environment \& Ecoplanning. 10 (2):525-528.

[12] Botros, G.A. (1970): The Length-Weight relationship \& coefficients of condition of Tilapia nilotica from lake mariut Egypt. - Review Zoology. Botany Africa :1-18.

[13] Boyd, C.E. (1979): Water quality in warm water fish ponds. - Auburn University, Agricultural Experiments station Auburn, Alabame USA : 79-98.

[14] Bush, R.M. \& Welch, E.B. (1972): Plankton association and related factors in hypertrophic lake. - Water, Air, Soil Pollution. 1:257-274.

[15] Datta, T. S .Acharya, \& Das, M.K. (2005): Impact of water quality on the stress physiology of cultured Labeo rohita. - Journal of Environmental Biology.26(3):582-592.

[16] Desikachary,T.V. (1959): Cyanophyta - Indian Council of Agricultural Research New Delhi.

[17] Dhanapathi, M.V.S.S.S. (2000): Taxonomic notes on the Rotifers from India (from 18892000). - Indian Association of Aquatic Biologists (IAAB) Publication No. 10.

[18] Doha, S \& Dewan, S. (1967): Studies on the Biology of Tilapia. (Tilapia mossambica peters). Length weight relationship and condition factors. - Pakisthan Journal of Science 19 (1\&2):23-28.

[19] Edmondson, W.T. (1959): Freshwater Biology - Rd., Edmondson, W.T. $2^{\text {nd }}$ edn., John Wiley \& Sons. Inc. Newyork.

[20] Goldman, C.R. \& Home, A.J. (1983): Limnology. - Pub. Mc Graw Hill, Inc. Japan.

[21] Gonzalves, E.A. (1981): Oedogoniales. - Indian Council of Agricultural Research New Delhi 1-757.

[22] Hart Paul, J.B., Reynolds, J.D. (2002): Handbook of fish biology \& fisheries. -Blackwell Publishing. U.K. 
[23] Haynes, R. (1982): Environmental science methods. - Chapman \& Hall, London, New York.

[24] Hile, R (1936): Age and growth of Cisco, leucichthys artedi (Le Sucer).In the lakes of the north estran highlands - Winconsin.Bull US..Burg fish 48: 311-317.

[25] Hutchinson,G.E. (1957): A treatise on Limnology: Geography, physics and chemisty. Vol. I 1015 PP. John Wiley \& sons, Inc. USA, (New York).

[26] Iwama, G.K., Vijayan, M.M., Morgan, J.D. (2000): The stress response in fish. Icthyology, Recent Research Advances 453 PP. Oxford \& IBH publishing co, Pvt. Ltd, N.Delhi.

[27] Jhingran, V.G. (1952): General length weight relationship of three major carps of India. Proc.Nat,Inst.Sci.India 18B, (5) :449-460.

[28] Jhingran, V.G. (1968): The length- weight relationship and K factor of Gurdusia chapra (Hamilton) from the Ganga river system. - Proc. Nat. Acad. Sci. India. B 38: 3-4.

[29] Joseph K.B., Richard, W.S., Daniel E. T.(1993): An introduction to water chemistry in freshwater aquaculture. - NRAC Fact sheet : 170.

[30] Kaur,H., Bath, K.S., Mander, G., Jeerath, N.(2000): Physico-chemical status of Kanjli wetland (Punjab-India). - J. environment \& pollution. 7(1): 39-42.

[31] Khabade,S.A., .Mule, M.B., Sathe, S.S.(2002): Studies on physco-chemical parameters of lodhe water reservoir from Taraon Tahsil (Maharashtra). - Indian J. environ \& ecoplan. 6(2): 301-304.

[32] Le'Cren, E.D. (1951): The Length relationship and seasonl cycle in Gonad weight and condition of perch (perces fluvuotilis). - Journal of Animal ecology 21:210-219.

[33] Lewis,W.M., Morris, D.P. (1986): Toxicity of nitrite to fish: A Review. - Transactions of American fisheries society. 115: 183-195.

[34] Maitland, P.S., (1990): Biology of Fresh waters. - Chapman and Hall. USA.

[35] Martin, W.B., Lutz, G.C., Robert, M.D. (1994): Algae blooms in commercial fish production ponds. - Southern regional aquaculture center (SRAC). SRAC publication No 466. United states Department of aquaculture.

[36] Martin, W.R. (1949): The mechanics of the environmental control of the body form in the fishes. - Univ.Toronto Stud. Biol. 58 Publ. Ont. Fish Res. Lab. 70:1-91.

[37] Moss, B. (1993): Ecology of Fresh waters - Man \& Medium. $2^{\text {nd }}$ Edition. Oxford Blackwell scientific building. London.

[38] Munawar, M. (1970): Limnological studies on freshwater ponds of Hyderabad, India. Hydrobiologia 31:101-128.

[39] Nwadario, C.S. (1985): The distribution \& food habits of dwarf African Cichlid pelvica chromis pulcher in the river Sombreiro, Nigeria. - Hydrobiologia. 121:157-184.

[40] Olatunde, A.A. (1983): The Length-Weight relationship and diets of Clarias Lazera in Zaria Nigeria. - Procedings of the $3^{\text {rd }}$ annual conference of the fisheries society of Nigeria. 183-192.

[41] Oslen,R.D., Sommerfeld, M.R. (1977): The physico-chemical limnology of a desert reservoir. - Hydrobiologia. 55 : 117-129.

[42] Palmer,C.M. (1980): Algae and water pollution. - Castle House Publication, USA.

[43] Pande,K.S., Sharma, S.D. (1999): Studies on water quality index for Ramaganga river at Moradabad - UP. Poll Res. 18 (3): 327-333.

[44] Patgiri,A., Goswami., M.M., Kar, D., Barbhuiya., M.H. (2001): Comparative Study on Length-Weight relationship and relative Condition factors in major and exotic carps in ponds of Huha. - Indian Journal of Environment \& Ecoplan. 5 :179-180.

[45] Prescott, G.W. (1982): Algae of the western great lakes area, Bishen Singh Mahendra pal Singh Dehradun - India. 31. P 1-997. Publishing house, New Delhi.

[46] Prinsloo, J.F., Schoonbee, H.J. (1987): Growth of Chinese grass-carp Ctenopharyngdon idella fed on cabbage wastes and kikyu grass. - Water. S.A., 13(2): 125-132

[47] Quadri, M.Y., Mir, S. (1980): Length-Weight relationship of Oreinus plangiostomus (MCCL). - Geobios. 7:158-159. 
[48] Raghavendra., Hosmani, S.P. (2002): Hydrobiological Study of Mandakally lake, A polluted waterbody at Mysore. - Nature Environment \& Pollution Technology. 1(3): 291293.

[49] Rekhow, K.H., Robert, W.B., Thomas, B.S., David, V., Udithwood., J. (1987): Empherical models of fish response to lake acidification. - Canadian Journal of Fish, Aquatic science. 44 (8): 1432-1442.

[50] Saxena,S.B., Adoni, A.D., (1973): Diurnal variation in Sagar lake, Sagar (India) 1. Studies in deep water areas. - Hydrobiol. 43: 435-443.

[51] Schroeder,G.L. (1980): Fish farming in manure loaded ponds. - Procedings ICLARMSEARCA conference in integrated agriculture and aquaculture farming systems. ICLARM procedings, 4: 73-86.

[52] Sharma, A., Chandrashekhar Gupta, R.T., Prabhu.,H.V. (1999): Studies on Zooplankton Community of Tungabhadra river in Karnataka receiving domestic sewage from Harihar region. - Journal of Environment and pollution. 6(2\&3): 161-166.

[53] Sharma,R.K., Rathore, V. (2000): Pollution ecology with refrence to commercially important fisheries prospect in a rural based water body : The lake Sarsai Nawar, Etawah in U.P. (India). - Pollution Research. 19(4): 641-644.

[54] Siddique, A.D. (1977): Reproductive biology, Length-Weight relationship \& relative condition of Tilapia Leucostica in lake Navaishe Kenya. - Journal of Fish biology. 10: 251-260.

[55] Singh, D.F., Rai, M.K. (1984): Studies on the ecology of engineering college lake of Jabalpur (M.P.). - J. Env. Biol. 5(3) : 165-168.

[56] Smart, G.R. (1978): Investigations of toxic mechanisms of ammonia to fish gas exchange in rainbow trout (Salmo gairdneri) exposed to acutely lethal concentrations. - Journal of Fish biology. $12: 93$.

[57] Solanki, R., Mandloi, A .K., Dubey, K.K. (2004): Length-Weight relationship \& condition factor in cirrhinus mrigala (Ham.) from a pond in Jabalpur. - National Journal of Life Sciences. 1(1): 101-106.

[58] Sreenivasan, A. (1965): Limnology \& productivity of tropical upland impoundments in Nilgiris, Madras state India. - Phycos. 7(1) : 146-160.

[59] Staggis, M.D., Otis, K.J. (1996): Factors affecting first years growth of fishes in lake Winnebago. - Wisconsin. N.A.M.J.Fish manage.16(3): 608-618.

[60] Sukumaran., .Das, A.K. (2005): Limnology \& fish production efficiency of selected reservoirs of Karnataka. - Indian Journal of Fish. 52(1): 47-53.

[61] Tesch,F.W. (1968): Age and growth. - In: "Methods for assesment of fish production in fresh waters" (ed. W. E. Ricker). IBP Handbook No.3, Blckwell, London.

[62] Tilak, K.S., Vardhan, K.S., Sumankumar, B. (2005): The effect of Ammonia, Nitrite and Nitrate on the oxygen consumption of the fish Ctenopharyngodon idella. - Journal of aquatic Biology.,Vol. 20(1): 117-122.

[63] Train \& Russel. (1979): Quality criteria for water - Castle house publications Ltd., 256 PP.

[64] Verma, M. (1967): Diurnal variation in a fish pond in Sioni, India. - Hydrobiol., 43: 453543.

[65] Welch,P.S. (1952): Limnology - 538 PP. Mc Graw-Hill Book co. Inc. USA.

[66] Yousuf, A.R., Mustafa Shah, G., Qadri, M.Y. (1986): Limnological aspects of Miragund wetland. - Geobios New Reports, 5: 27-30.

[67] Zweig Ronaldo, D. (1989): Evolving water quality in a common carp \& blue tilapia high production pond. - Hydrobiologia 171: 11-21. 